\title{
NUMERICAL STUDY ON THE PERFORMANCE OF 2-D UGRINSKY WIND TURBINE MODEL
}

\author{
LUKE SAKAMOTO, TOMOHIRO FUKUI \& KOJI MORINISHI \\ Department of Mechanical Engineering, Kyoto Institute of Technology, Japan
}

\begin{abstract}
Improving efficiency in vertical axis wind turbines (VAWTs), represented by the Savonius wind turbine, is desired with the increase in attention to renewable energy. The Ugrinsky wind turbine is a type of VAWT, which has a pair of blades consisting of a semicircle and a circular arc smoothly connected and is expected to have better performance than the Savonius wind turbine with producing positive torque at all angles in a rotation although there are few studies on this Ugrinsky wind turbine. Our prior research has shown that the maximum output power coefficient of the Ugrinsky wind turbine reaches $0.170,43.0 \%$ higher than that of the Savonius model $(0.110)$, and sustained its high-power coefficient in a wide range of tip speed ratios (TSR). This is due to both of the semicircular blades producing a positive torque during the advancing blade, whereas during the returning blade only the larger radius blade mainly produces a negative torque because of its overlapping layout. In this study, the dimensions of the two semicircles of the Ugrinsky wind turbine are optimised to obtain a higher power coefficient. The flow around the turbine was simulated by using the regularized lattice Boltzmann method. The virtual flux method was used to describe the shape of the turbine on Cartesian grids, and the multi-block method was used for the local fine grids around the turbine. The rotational speed of the turbine was maintained as a constant, and its performance was evaluated by the output power and torque coefficients. The results show that compared to the original Ugrinsky wind turbine model, the maximum power coefficient was improved by $1.5 \%$, and the average value of the power coefficient for neighbouring tip speed ratios was improved by $5.9 \%$.

Keywords: vertical axis wind turbine (VAWT), Ugrinsky wind turbine, Savonius wind turbine, dragtype wind turbine, power coefficient, torque coefficient.
\end{abstract}

\section{INTRODUCTION}

In recent years, wind energy has gained a lot of attention as a means of renewable energy resource to combat climate change and pollution. In the Sustainable Development Scenario presented by the International Energy Agency (IEA), wind energy provides $21 \%$ of electricity in 2040, yet as of 2018 this rate is merely 5\% [1]; hence further innovation in this field is required. Wind turbines can be classified into two categories depending on their rotational axes: horizontal axis wind turbines (HAWTs), and vertical axis wind turbines (VAWTs). The propeller wind turbine is considered to be the standard of HAWTs and is widely used in large-scale electricity generation due to its high output power coefficient [2]. Compared to HAWTs, VAWTs are often favoured in small-scale power generation and have the advantage of being omnidirectional (independent of wind direction) [3]. VAWTs can also be divided into drag-type and lift-type depending on the aerodynamic force that drives the rotation [2]. In general, lift-types are known to be more efficient than drag-types, though normally they generate low torque in low tip speed ratios (TSRs) and are consequently unsuitable for selfstart [4]. In contrast, the Savonius wind turbines, the standard of drag-type VAWTs, are known for (i) high start-up torque, (ii) no low-frequency noise pollution, (iii) simple and lowcost design and (iv) operation in a wide range of wind conditions compared to lift-type VAWTs [5], [6].

Advantages of being omnidirectional, (i), (ii) and (iv), make drag-type VAWTs an ideal option for installing wind turbines in urban environments, with increased interest in 
employing wind turbines in urban environments in recent years [7]-[9]. However, their output power coefficient is generally poor compared to that of the propeller type by less than half [10]. Therefore, a large variety of studies are directed towards finding a better design for VAWTs. One example of this is installing shields or plates in front of the turbine [11], [12]. However, installing obstacles for improving efficiency will lose the independence of the wind direction, and will require additional land area. Thus, modifying the blade shape is the most effective method for improving its efficiency while ensuring the independence of the wind direction. Zhang et al. [6] improved the power coefficient by $6 \%$ while simultaneously reducing the blade weight by $17.9 \%$, by using a quadratic polynomial curve on a blade shape of the traditional Savonius-style turbine. Roy and Ducoin [13] came up with a novel twobladed Savonius-style wind turbine and were able to improve the power coefficient by $32.1 \%$ compared to the traditional Savonius counterpart. Matsui et al. [14] added a set of sub blades to a two-blade VAWT similar to a Bach-type wind turbine and by optimising the position of them, the power coefficient reached $50.7 \%$ higher than that of the traditional Savonius turbine.

The Ugrinsky wind turbine is a type of VAWT, which has a pair of blades consisting of a semicircle and a circular arc that shares the same diameter as the turbine [15]. The turbine performs better than the traditional Savonius wind turbine by $43.2 \%$; moreover, producing positive torque at all angles throughout a cycle at $\lambda=0.5$ [16]. However, so far, there are few studies on this turbine, and no usage example as an electrical generation was found. With its high efficiency compared to the Savonius model, this turbine can potentially be a viable option for the future installation of VAWTs. In this paper, the optimisation for the two circle's dimensions is conducted to improve the power coefficient.

\section{NUMERICAL METHODS}

The regularized lattice Boltzmann method (RLBM) is used as a governing equation in this paper [17], [18]. The virtual flux method is used to describe the blades of the turbines on a Cartesian grid [19]. The multi-block method is used for the locally fine grids around the turbines [20]. The simulation was conducted in 2-D, which is known to give acceptable results and shares the performance characteristics with 3-D simulation [3], [5].

\subsection{Regularized lattice Boltzmann method}

The RLBM is used as a governing equation for the 2D 9-velocity (D2Q9) model for the fluid analysis. This method is designed to reduce memory usage and simulate flow at high Reynolds numbers without compromising accuracy compared to the lattice Boltzmann method [21].

The distribution function $f_{\alpha}$ is expressed as

$$
f_{\alpha}=w_{\alpha}\left(a_{0}+b_{i} e_{\alpha i}+c_{i j} e_{\alpha i} e_{\alpha j}\right),
$$

where $w_{\alpha}$ is the weight coefficient defined by the lattice speed model $\left(w_{0}=4 / 9, w_{1-4}=\right.$ $1 / 9, w_{5-9}=1 / 36$ for the D2Q9 model). $a_{0}, b_{i}$ and $c_{i j}$ are determined to satisfy the following relationships:

$$
\begin{gathered}
\sum_{\alpha} f_{\alpha}=\rho, \\
\sum_{\alpha} e_{\alpha i} f_{\alpha}=\rho u_{i}, \\
\sum_{\alpha} e_{\alpha i} e_{\alpha j} f_{\alpha}=\frac{c^{2}}{3} \rho \delta_{i j}+\rho u_{i} u_{j}+\Pi_{i j}^{\mathrm{neq}},
\end{gathered}
$$


where $\rho$ is the fluid density, $u_{i}$ is the fluid velocity component, $c$ is the lattice speed defined as $c=\delta x / \delta t, \delta_{i j}$ is the Kronecker delta, and $\Pi_{i j}^{\text {neq }}$ is the non-equilibrium part of the stress tensor. Then, the equilibrium distribution function $f_{\alpha}^{\text {eq }}$, and the non-equilibrium part of the distribution function $f_{\alpha}^{\text {neq }}$ is expressed as follows:

$$
\begin{gathered}
f_{\alpha}^{\mathrm{eq}}=w_{\alpha} \rho\left[1+\frac{3\left(e_{\alpha i} u_{i}\right)}{c^{2}}+\frac{9\left(e_{\alpha i} u_{i}\right)^{2}}{2 c^{4}}-\frac{3\left(u_{i} u_{i}\right)}{2 c^{2}}\right], \\
f_{\alpha}^{\text {neq }}=\frac{9 w_{\alpha}}{2 c^{2}}\left(\frac{e_{\alpha i} e_{\alpha j}}{c^{2}}-\frac{1}{3} \delta_{i j}\right) \Pi_{i j}^{\mathrm{neq}},
\end{gathered}
$$

where the equilibrium distribution function $f_{\alpha}^{\text {eq }}$ is expressed by approximating the Maxwell equilibrium distribution function to the quadratic term. Lastly, the time evolution equation in the regularized lattice Boltzmann equation is expressed as

$$
f_{\alpha}\left(t+\delta t, \boldsymbol{x}+\boldsymbol{e}_{\alpha} \delta t\right)=f_{\alpha}^{\mathrm{eq}}(t, \boldsymbol{x})+\left(1-\frac{1}{\tau}\right) f_{\alpha}^{\mathrm{neq}},
$$

where $\tau$ is the relaxation time.

\subsection{Virtual flux method}

The virtual flux method was used to describe the wind turbine shape in a Cartesian grid. This method was chosen due to its simplicity in the algorism with no iterative calculations. Moreover, this method can describe the pressure field around an object more accurately compared to other immersed boundary methods for the same grid resolution. Fig. 1 shows the schematic view of the virtual boundary points. With the lattice Boltzmann method using D2Q9model, virtual boundary points are set at the intersections of discrete velocities in eight directions and the object surface.

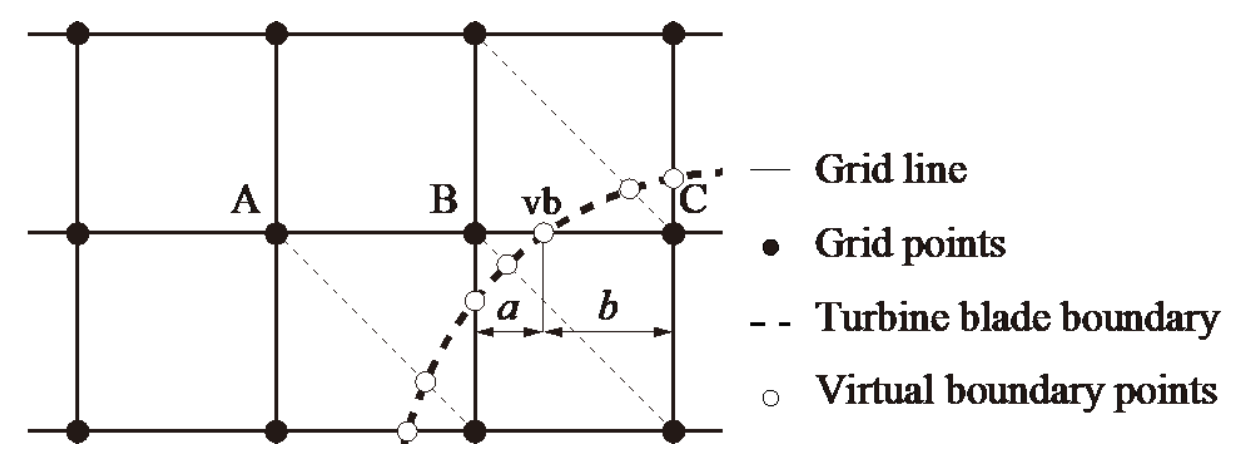

Figure 1: Schematic view of the turbine blade boundary on a Cartesian grid.

First, the physical quantity on the virtual boundary $q_{\mathrm{vb}}$ is considered. The no-slip boundary condition $\left(\boldsymbol{u}_{\mathrm{vb}}=\boldsymbol{u}_{\mathrm{wall}}\right)$ is applied for the velocity of the virtual boundary $\boldsymbol{u}_{\mathrm{vb}}$, where $\boldsymbol{u}_{\text {wall }}$ is the velocity on the wall surface of the turbine blade. The Neumann boundary condition $\left(\partial p_{\mathrm{vb}} / \partial \boldsymbol{n}=0\right)$ is applied for the pressure of the virtual boundary $p_{\mathrm{vb}}$, where $\boldsymbol{n}$ is the normal vector on the virtual boundary wall, providing the approximate pressure condition on the surface.

Next, as shown in Fig. 2, the case where the distribution function at point " $\mathrm{C}$ " moves to point " $\mathrm{B}$ " is considered; however, this movement is obstructed by the virtual boundary. 
Therefore, the virtual distribution function $f_{\alpha, \mathrm{vb}}$ will be calculated at point "vb" from the distribution function $f_{\alpha, \mathrm{B}}$ and the equilibrium distribution function $f_{\alpha, \mathrm{B}}^{\mathrm{eq}}$ as follows:

$$
f_{\alpha, \mathrm{vb}}=f_{\alpha, \mathrm{vb}}^{\mathrm{eq}}\left(u_{\mathrm{vb}}, p_{\mathrm{vb}}\right)+\left(f_{\alpha, \mathrm{B}}-f_{\alpha, \mathrm{B}}^{\mathrm{eq}}\right) .
$$

Then, the virtual distribution function $f_{\alpha, \mathrm{C}}^{*}$ and the virtual equilibrium distribution function $f_{\alpha, \mathrm{C}}^{\mathrm{eq} *}$ at point " $\mathrm{C}$ " are calculated by extrapolation using internal division ratios $a$ and $b$.

$$
\begin{aligned}
f_{\alpha, \mathrm{C}}^{*} & =\frac{a+b}{a} f_{\alpha, \mathrm{vb}}-\frac{b}{a} f_{\alpha, \mathrm{B}}, \\
f_{\alpha, \mathrm{C}}^{\mathrm{eq} *} & =\frac{a+b}{a} f_{\alpha, \mathrm{vb}}^{\mathrm{eq}}-\frac{b}{a} f_{\alpha, \mathrm{B}}^{\mathrm{eq}} .
\end{aligned}
$$

Finally, the distribution function a point " $\mathrm{B}$ " for the next time step $f_{\alpha, \mathrm{B}}$ is obtained from $f_{\alpha, \mathrm{C}}^{*}$ and $f_{\alpha, \mathrm{C}}^{\mathrm{eq} *}$ as follows:

$$
f_{\alpha, \mathrm{B}}=f_{\alpha, \mathrm{C}}+\frac{1}{\tau}\left(f_{\alpha, \mathrm{C}}^{\mathrm{eq}}-f_{\alpha, \mathrm{C}}^{*}\right)
$$

\subsection{Computational models}

In this research, the flow around the Ugrinsky wind turbine with various dimensions is simulated and evaluated with the power coefficients and the torque coefficients. Fig. 2(a) shows the schematic view of the Ugrinsky wind turbine with the original dimensions, where $D$ is the diameter of the turbine circle (drawn with a dashed line), $S$ is the diameter of "Segment 1", and $L$ is the radius of "Segment 2". In addition, the radius of the turbine $R$ (= $D / 2)$ is used to describe the dimension of $L$ in this paper. The original dimensions of the Ugrinsky wind turbine are $(S, L)=(0.40 D, 1.0 R)$. Fig. 2(b) shows the Savonius model used in verification, where $l$ is the blade length and $e$ is the overlap ratio, which is set to $0.2 l$.
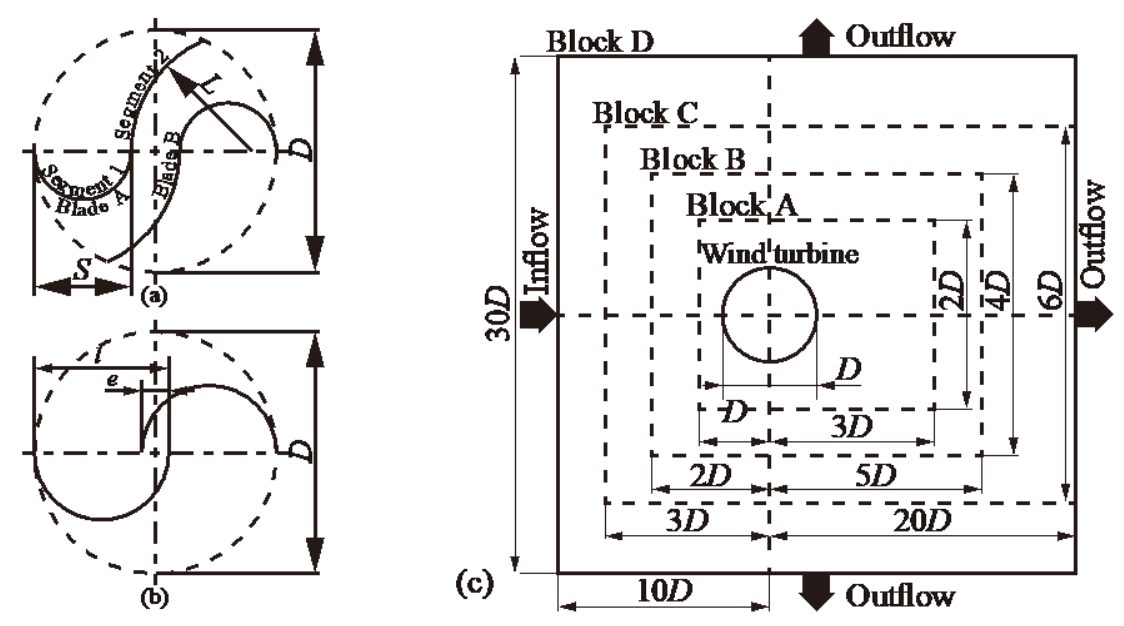

Figure 2: Schematic view of: (a) The Ugrinsky wind turbine at $0^{\circ}$; (b) The Savonius wind turbine at $0^{\circ}$; and (c) The multi-block model used in the simulation.

Fig. 2(c) shows the schematic view of the multi-block model for the simulation. The computational domain was set as $30 D \times 30 D$ using the multi-block method and the 
coordinates for the centre of the turbine was set at $(10 D, 15 D)$. The convective flow condition was applied at the right-side outflow of the domain [22]. The velocity and the pressure gradients are both equal to 0 at the upper and lower sides of the domain. The Reynolds number is set as 1,000 , based on the turbine's diameter. The rotational speed was set as a constant value to satisfy the following TSRs: $\lambda=0.3,0.4, \ldots, 0.9$. To reduce the calculation cost, multiple TSRs are calculated in sequence in this simulation. For example, for the case of the Segment 1 optimisation, $\lambda=0.7$ is set as an initial TSR and nine cycles are performed in total, and the last three cycles are used for evaluation. Next, the blade rotational speed gradually decreases to meet $\lambda=0.6$ at the beginning of the 10 th cycle. Six cycles are performed including the last three cycles for evaluation for $\lambda=0.6$. Then, the TSR is decreased to $\lambda=0.5$ and calculation will be continued in the same way as $\lambda=0.6$. This method is more effective than calculating each TSR individually since each calculation requires the preparation cycles for eliminating the initial condition at the beginning. The output of the evaluation cycles is averaged for the power coefficient values and the instantaneous torque plots.

The torque coefficient $C_{Q}$, the power coefficient $C_{P}$, and the TSR $\lambda$ are defined as follows:

$$
\begin{gathered}
C_{Q}=\frac{T}{\frac{1}{2} \rho U^{2} R A}, \\
C_{P}=\frac{T \cdot \omega}{\frac{1}{2} \rho U^{3} R A}=C_{Q} \cdot \lambda, \\
\lambda=\frac{R \omega}{U},
\end{gathered}
$$

where $T$ is the torque, $U$ is the characteristic velocity, $\omega$ is the angular velocity, and $A$ is the swept area. The optimal shape is selected by the maximum power coefficient value and the average value of the power coefficient for neighbouring TSRs. For example, the average value of the power coefficient for neighbouring TSRs at $\lambda=0.6$ is the average value of the power coefficient of $\lambda=0.5,0.6$, and 0.7 . By doing so, the TSR fluctuation of turbine occurs in experimental test due to non-uniform flow or changes in torque values with angle can be taken into consideration.

\subsection{Verification}

A verification study was conducted on a Savonius turbine shown in Fig. 2(b) for Reynolds number $R e=500$ at the TSR $\lambda=0.8$. The test was conducted with four different grids for the characteristic length $(D=256,360,512,724$ cells). Fig. 3 shows the torque coefficient of the 6th rotation, and Table 1 shows the results of the averaged torque coefficient. The transition of the torque coefficient value and the angle of rotation at which the maximum and minimum values are obtained show the same trend as other studies [5], [6]. The number of grids required for the characteristic length is proportional to $R e^{1 / 2}$ times by the boundary layer thickness theory [23]. The average torque coefficient for $D=256$ cells shows less than $3 \%$ of error compared to $D=724$ cells at $R e=500$; therefore, $D=360$ cells were selected for $R e=1,000$ condition.

\section{RESULTS AND DISCUSSION}

In this section, the effects of optimisation on Segment 1 and Segment 2 are discussed. The performance of the turbines is described in torque and power coefficients, and the pressure coefficient was used in the pressure contour diagram for visualisation. 


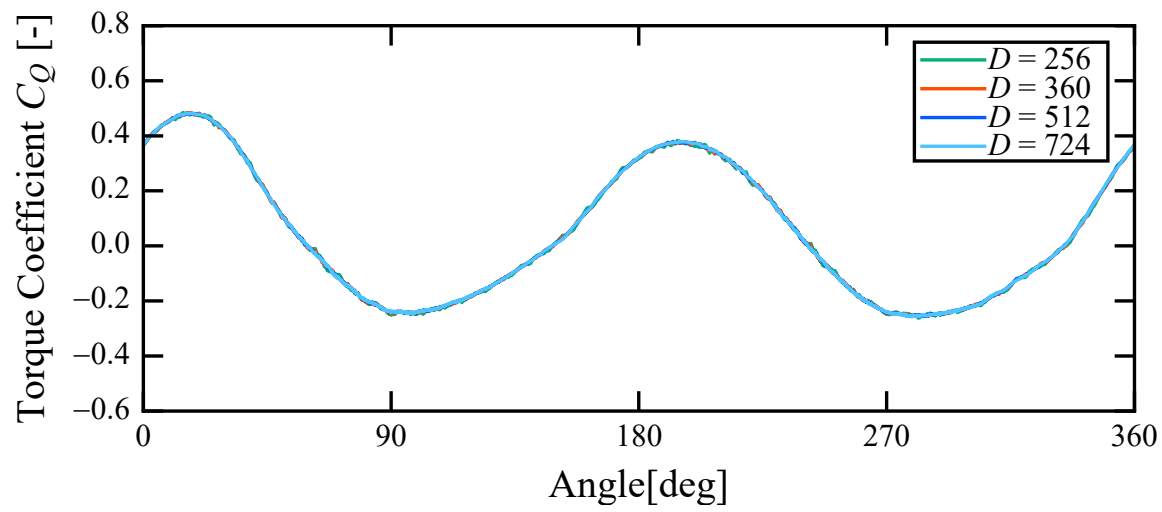

Figure 3: Torque coefficient values for different resolutions.

Table 1: Averaged torque coefficient values for different resolutions.

\begin{tabular}{cc}
\hline $\begin{array}{c}\text { Number of grids for the } \\
\text { characteristic length }\end{array}$ & Averaged torque coefficient \\
\hline 256 cells & 0.042855 \\
360 cells & 0.043666 \\
512 cells & 0.044075 \\
724 cells & 0.044173 \\
\hline
\end{tabular}

\subsection{Optimisation for Segment 1}

Fig. 4 shows the comparisons of the average power coefficients of different diameters for Segment 1 , and Table 2 highlights the maximum average power coefficients and the averaged power coefficient values of neighbouring TSRs. The highest power coefficient was achieved by $S=0.40 D$, the original Ugrinsky wind turbine model, and $S=0.35 D$ both at $\lambda=0.5$. However, while the $S=0.35 D$ model maintains a high power coefficient over a wide range of TSRs, the $S=0.40 D$ model decreases its power coefficient by a larger degree than the $S=0.35 D$ model above $\lambda=0.5$. The $S=0.35 D$ model was selected for optimisation on Segment 2 judging from the maximum power coefficient at $\lambda=0.5$ and the ability to maintain its high torque coefficient at $\lambda=0.5-0.7$.

To further analyse the effect of Segment 1 on the Ugrinsky wind turbine, the instantaneous torque coefficient is plotted for $S=0.30 D, 0.35 D$ and $0.40 D$ models for $\lambda=0.6$ in Fig. 5 . The torque coefficient plots were divided into Segment 1 and Segment 2 for easier understanding. Each corresponding pressure contour addressed in Fig. 5 is plotted in Fig. 8.

At $50^{\circ}$, there is no improvement in torque with the increase in $S$ beyond $S=0.35 \mathrm{D}$ for Segment 1 (in Fig. 5 and Fig. 8 (A)). At $330^{\circ}, S=0.30 D$ and $0.35 D$ produces smaller negative torque compared to $S=0.40 D$ on Segment 1 . This is due to the narrow wind-receiving area of Segment 1 during the return blade period; furthermore, positive torque was produced due to the negative pressure area created on the convex part of Segment 1 for $S=0.30 D$ and $0.35 D$ (in Fig. $5(\mathrm{~B})$ ). 


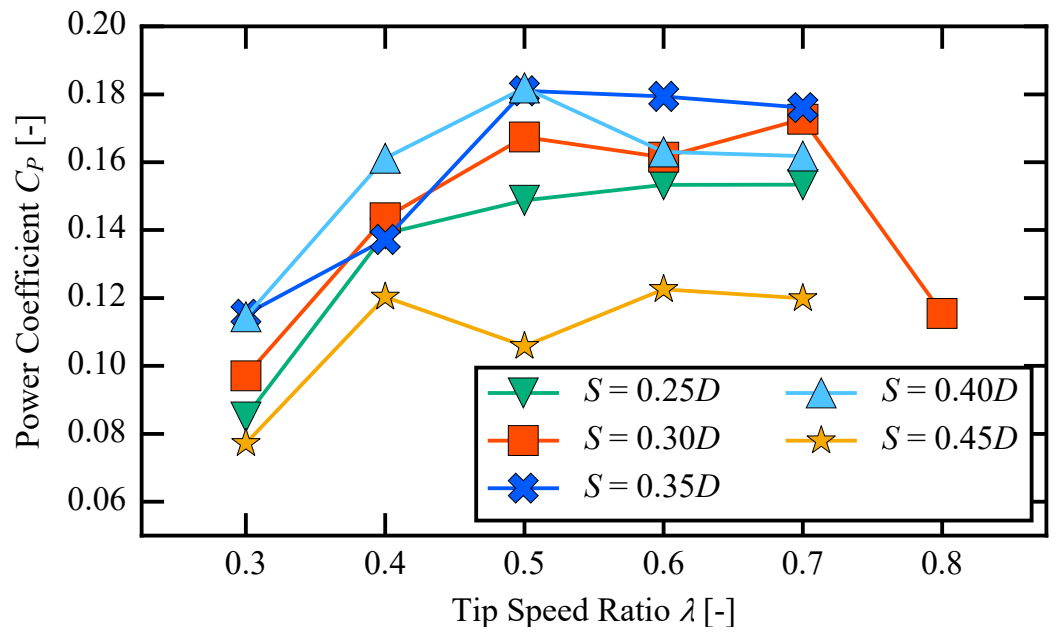

Figure 4: Power coefficient values of the Ugrinsky wind turbine models with different diameters for Segment 1 in dynamic simulation. $C_{P}$ at $\lambda=0.8$ was calculated only for $S=0.30 D$ to display its peak TSR.

Table 2: Maximum power coefficient values and the average value of the power coefficient for neighbouring TSRs of different diameters $S$ for Segment 1.

\begin{tabular}{ccc}
\hline$S$ & Maximum $C_{P}$ & Average $C_{P}$ \\
\hline $0.25 D$ & $0.153(\lambda=0.6)$ & $0.152(\lambda=0.5-0.7)$ \\
$0.30 D$ & $0.173(\lambda=0.7)$ & $0.167(\lambda=0.5-0.7)$ \\
$0.35 D$ & $0.181(\lambda=0.5)$ & $0.179(\lambda=0.5-0.7)$ \\
$0.40 D$ & $0.182(\lambda=0.5)$ & $0.169(\lambda=0.5-0.7)$ \\
$0.45 D$ & $0.123(\lambda=0.5)$ & $0.116(\lambda=0.4-0.6)$ \\
\hline
\end{tabular}

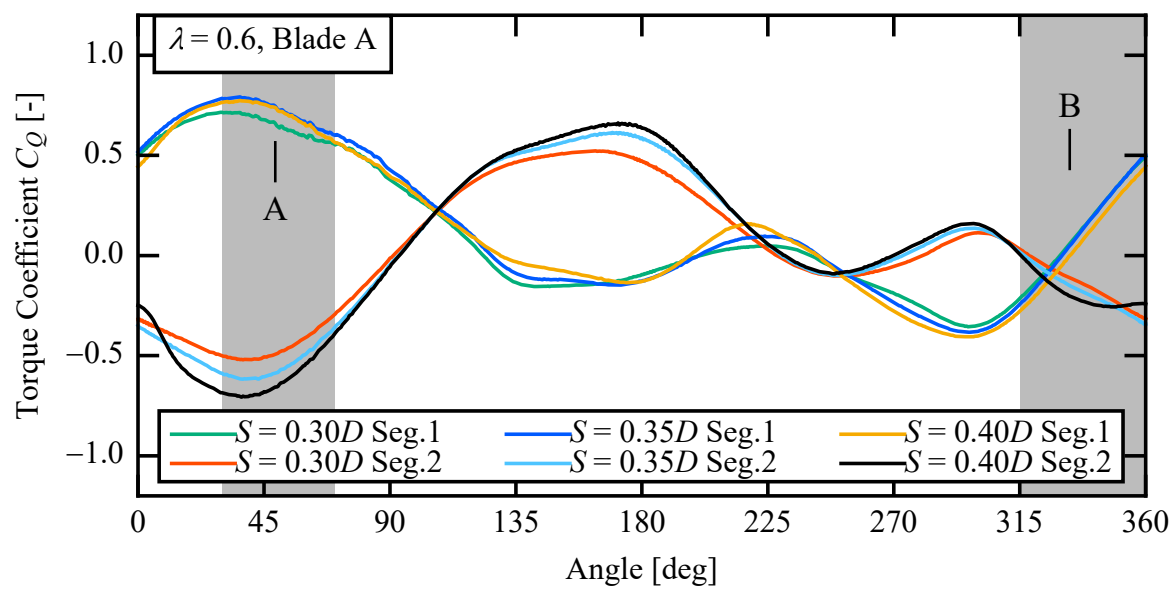

Figure 5: Comparison of the torque coefficient at different rotor angle for $S=0.30 D$, $0.35 D$ and $0.40 D$ at $\lambda=0.6$. 


\subsection{Optimisation for Segment 2}

Fig. 6 shows the comparison of the average power coefficients of different radii for Segment 2 , and Table 3 highlights the maximum average power coefficients and the average value of the power coefficient for neighbouring TSRs. Overall, by changing $L$ from the original value $(L=1.0 R)$, the TSR for the peak power coefficient value increases. Notably, the $L=1.1 R$ and $1.2 R$ models show similar characteristics and both models hit a low power coefficient value at $\lambda=0.6$ compared to the other models.

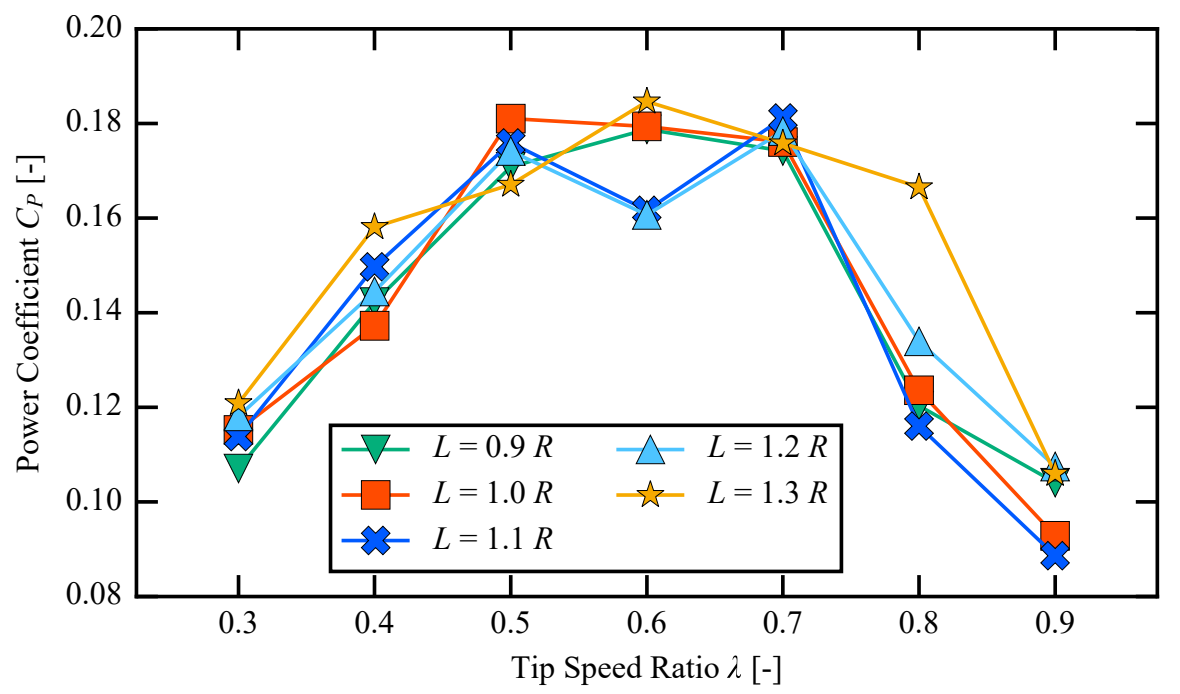

Figure 6: Power coefficient values of the Ugrinsky wind turbine model with different radii for Segment 2 in dynamic simulation.

Table 3: Maximum power coefficient values and the average value of the power coefficient for neighbouring TSRs of different radii $L$ for Segment 2.

\begin{tabular}{ccc}
\hline$L$ & Maximum $C_{P}$ & Average $C_{P}$ \\
\hline $0.9 R$ & $0.179(\lambda=0.6)$ & $0.175(\lambda=0.5-0.7)$ \\
$1.0 R$ & $0.181(\lambda=0.5)$ & $0.179(\lambda=0.5-0.7)$ \\
$1.1 R$ & $0.181(\lambda=0.7)$ & $0.173(\lambda=0.5-0.7)$ \\
$1.2 R$ & $0.178(\lambda=0.7)$ & $0.171(\lambda=0.5-0.7)$ \\
$1.3 R$ & $0.185(\lambda=0.6)$ & $0.176(\lambda=0.4-0.6)$ \\
\hline
\end{tabular}

To understand why the $L=1.1 R$ and $1.2 R$ models were inferior to $L=1.0 R$ or $1.3 R$ at $\lambda=0.6, L=1.0 R, 1.1 R$ and $1.3 R$ were chosen for the comparison in Fig. 7. As shown in Fig. 7 , the $L=1.1 R$ model has a different characteristic compared to others. In order to visualise the characteristic difference, the pressure distribution of the wake flow of the rotor for $100^{\circ}$ and $280^{\circ}$ for the $L=1.0 R, 1.1 R$ and $1.3 R$ models were shown in Fig. 8 (C) and (D), respectively. At $280^{\circ}$, it can be seen that the vortex detached from the tip of Blade A Segment 2 moves to the wake region regardless of $L$ (purple circle). On the other hand, at $100^{\circ}$, while $L=1.1 R$ model's vortex characteristic is the same as at $280^{\circ}$, the vortex generated by Blade 


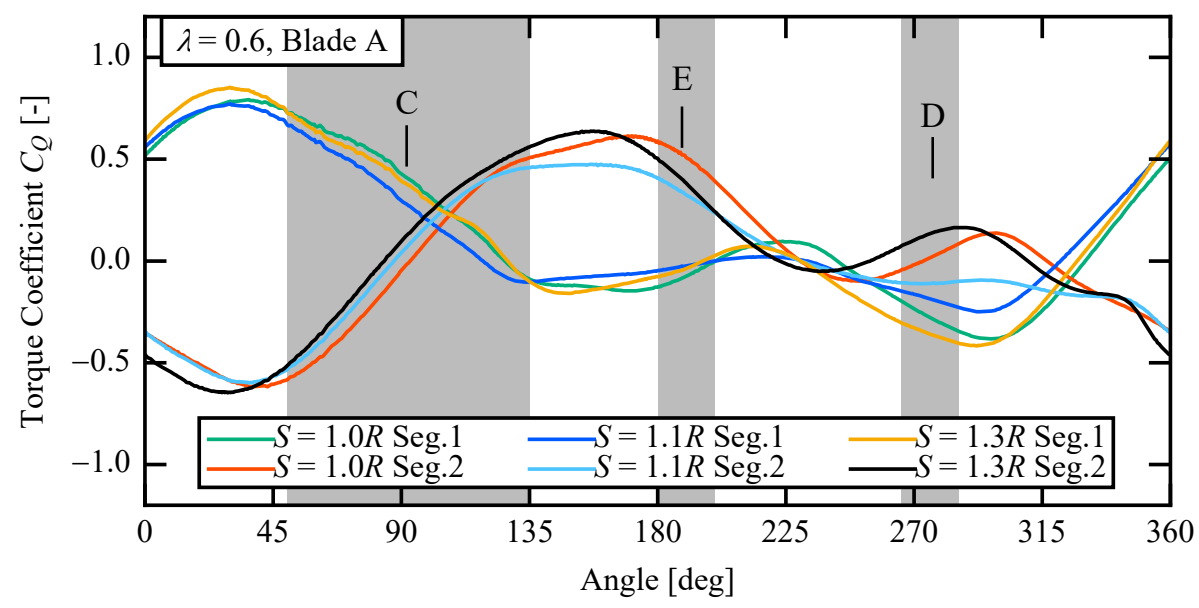

Figure 7: Comparison of the torque coefficient at different rotor angles for $L=1.0 R, 1.1 R$ and $1.3 R$ at the tip speed ratio $\lambda=0.6$.

B Segment 2 remains attached and rotate with Blade B for $L=1.0 R$ and $1.3 R$ (red circle).Due to the fluid-structure difference outlined above, in the advancing blade region of Segment 1 on Blade A $\left(45-135^{\circ}\right)$, a large vortex is generated for $L=1.0 R$ and $1.3 R$ at the tip of Segment 1 (in Fig. 7 and $8(\mathrm{C})$, black circle). This vortex creates a low-pressure area on the convex side of Segment 1, consequently developing large torque on the blade. Likewise, a larger vortex is observed at the tip of Blade A, Segment 2 for $L=1.0 R$ and $1.3 R$ compared to $L=1.1 R$ at $190^{\circ}$, developing high torque on the blade for these models (in Fig. 7 and $8(\mathrm{E})$ ). Generally, the larger $L$ is, the greater the torque produced by the blades, due to the increase in arm length; however, the fluid-structure properties are more dominant than geometrical properties in $\lambda=0.6$, and therefore this phenomenon, where the $L=1.0 R$ model performs better than the $L=1.1 R$ model, was observed at this angle.

\section{CONCLUSIONS AND FUTURE WORK}

In this paper, the flow around the Ugrinsky wind turbine with various blade dimensions was simulated to investigate the optimal shape. For optimization, the optimal diameter of $S$ was first selected, and then the optimal radius of $L$ was selected through simulations. Maximum power coefficient and stability of power coefficient in neighbouring TSRs were employed for the evaluation. Based on the results in this paper, the following conclusions have been drawn.

1. The optimal parameters of the Ugrinsky wind turbine were $S=0.35 D, L=1.0 R$ for stable power coefficient at $\lambda=0.5-0.7 ; S=0.35 D, L=1.3 R$ for maximum power coefficient at $\lambda=0.6$.

2. Compared to the original Ugrinsky wind turbine model $(S=0.40 D, L=1.0 R)$, the maximum power coefficient was improved by $1.5 \%$.

3. Compared to the original Ugrinsky wind turbine model $(S=0.40 D, L=1.0 R)$, the average value of the power coefficient for neighbouring tip speed ratios was improved by $5.9 \%$.

4. The presented optimum Ugrinsky models offer a viable option for future installation of VAWTs. 


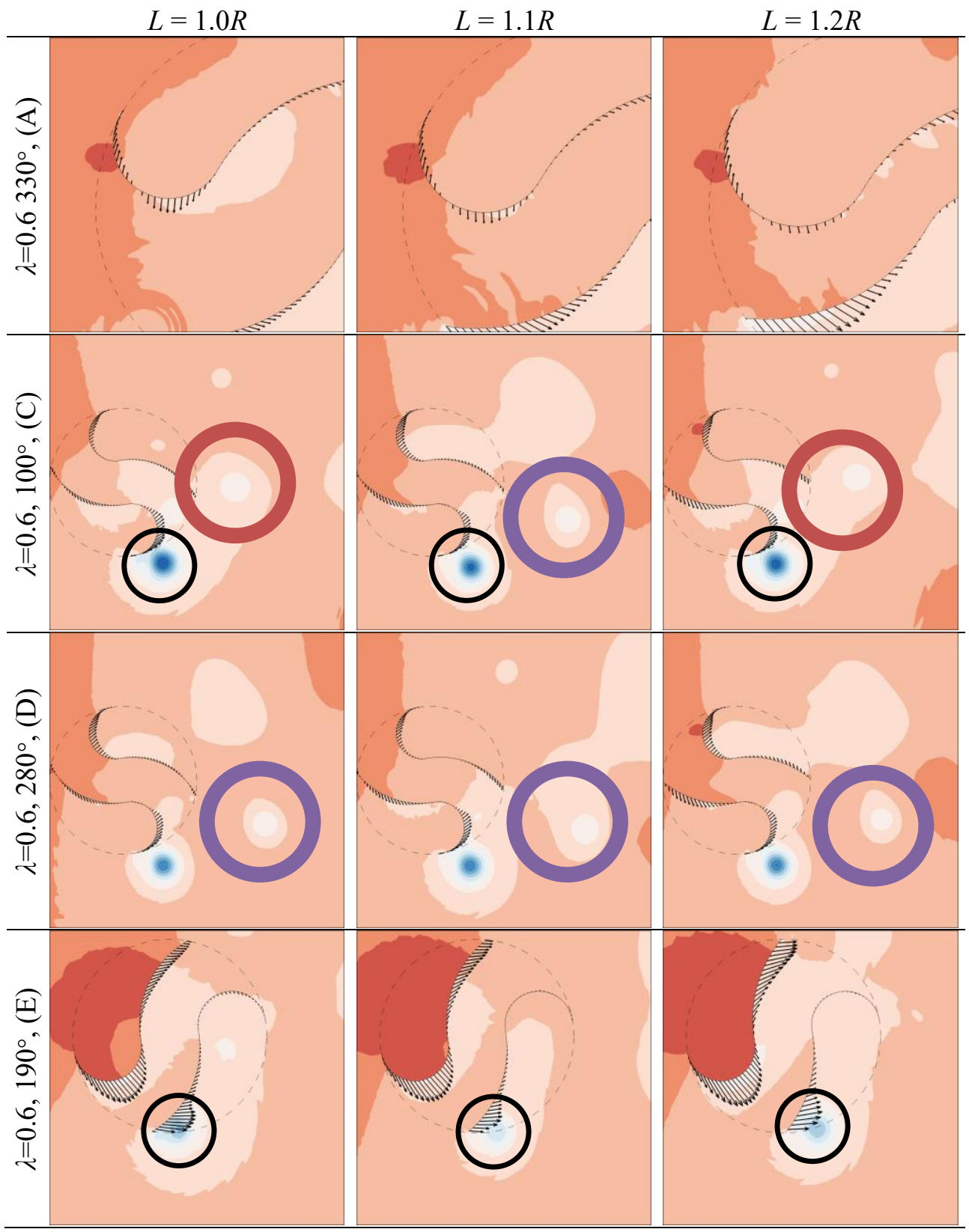

Figure 8: Pressure coefficient distributions around the Ugrinsky models for different Segment 2 radii. The black vectors are representing the torque generated on the blades. 
It should be noted that the blade length is not considered during this optimisation. Thus, in order to reduce the weight and the moment of inertia, the proposed wind turbine shape should be optimised in terms of blade length to further increase efficiency.

\section{REFERENCES}

[1] International Energy Agency (IEA), World Energy Outlook 2019, IEA: Paris, 2019.

[2] Jang, H., Kim, D., Hwang, Y., Paek, I., Kim, S. \& Baek, J., Analysis of Archimedes spiral wind turbine performance by simulation and field test. Energies, 12(24), p. 4624, 2019. https://doi.org/10.3390/en12244624.

[3] Roy, S. \& Saha, U.K., Review on the numerical investigations into the design and development of Savonius wind rotors. Renewable and Sustainable Energy Reviews, 24, pp. 73-83, 2013. https://doi.org/10.1016/j.rser.2013.03.060.

[4] Mitchell, S., Ogbonna, I. \& Volkov, K., Improvement of self-starting capabilities of vertical axis wind turbines with new design of turbine blades. Sustainability, 13(7), p. 3854, 2021. https://doi.org/10.3390/su13073854.

[5] Tian, W., Song, B., Van Zwieten, J.H. \& Pyakurel, P., Computational fluid dynamics prediction of a modified Savonius wind turbine with novel blade shapes. Energies, 8(8), pp. 7915-7929, 2015. https://doi.org/10.3390/en8087915.

[6] Zhang, B., Song, B., Mao, Z., Tian, W., Li, B. \& Li, B., A novel parametric modeling method and optimal design for Savonius wind turbines. Energies, 10(3), p. 301, 2017.

[7] Alaimo, A., Esposito, A., Milazzo, A., Orlando, C. \& Trentacosti, F., Slotted blades Savonius wind turbine analysis by CFD. Energies, 6(12), pp. 6335-6351, 2013.

[8] Chakroun, Y. \& Bangga, G., Aerodynamic characteristics of airfoil and vertical axis wind turbine employed with Gurney flaps. Sustainability, 13(8), p. 4284, 2021.

[9] Müller, G., Chavushoglu, M., Kerri, M. \& Tsuzaki T., A resistance type vertical axis wind turbine for building integration. Renewable Energy, 111, pp. 803-814, 2017. https://doi.org/10.1016/j.renene.2017.05.017.

[10] Ushiyama, I., Nagai, H. \& Shinoda, J., Experimentally determining the optimum design configuration for Savonius rotors. Transactions of the Japan Society of Mechanical Engineers Series B, 52(480), pp. 2973-2981, 1986.

[11] Mohamed, M., Gábor, J., Elemér, P. \& Dominique, T., Optimization of Savonius turbines using an obstacle shielding the returning blade. Renewable Energy, 35(11), pp. 2618-2626, 2010.

[12] El-Askary, W.A., Nasef, M.H., Abdel-Hamid, A.A. \& Gad, H.E., Harvesting wind energy for improving performance of Savonius rotor. Journal of Wind Engineering and Industrial Aerodynamics, 139, pp. 8-15, 2015.

[13] Roy, S. \& Ducoin, A., Unsteady analysis on the instantaneous forces and moment arms acting on a novel Savonius-style wind turbine. Energy Conversion and Management, 121, pp. 281-296, 2016.

[14] Matsui, T., Fukui, T. \& Morinishi, K., Computational fluid dynamics on a newly developed Savonius rotor by adding sub-buckets for increase of the tip speed ratio to generate higher output power coefficient. Journal of Fluid Science and Technology, 15(2), 0009, pp. 1-16, 2020.

[15] Kazhinsky, B.B., Low-Capacity Free-Flow Hydroelectric Power Plants, Gosenergoizdat: Moscow, pp. 30-32, 1950. (In Russian.)

[16] Sakamoto, L., Fukui, T. \& Morinishi, K., Performance evaluation of Ugrinsky wind turbine using numerical simulation. Proceedings of the Fluids Engineering Conference, 2020. (In Japanese.) https://doi.org/10.1299/jsmefed.2020.OS09-04. 
[17] Izham, M., Fukui, T. \& Morinishi, K., Application of regularized lattice Boltzmann method for incompressible flow simulation at high Reynolds number and flow with curved boundary. Journal of Fluid Science and Technology, 6(6), pp. 812-822, 2011.

[18] Morinishi, K. \& Fukui, T., An Eulerian approach for fluid-structure interaction problems. Computers and Fluids, 65, pp. 92-98, 2012.

[19] Tanno, I., Morinishi, K., Matsuno, K. \& Nishida, H., Validation of virtual flux method for forced convection flow. JSMR International Journal Series B, 46(4), pp. 11411148, 2006.

[20] Yu, D., Mei, R. \& Shyy, W., A multi-block lattice Boltzmann method for viscous fluid flows. International Journal for Numerical Methods in Fluids, 39, pp. 99-120, 2002.

[21] Chen, S. \& Doolen, G.D., Lattice Boltzmann method for fluid flows. Annual Review of Fluid Mechanics, 30, pp. 329-364, 1998.

[22] Tsutahara, M. \& Hiraishi, M., Study of outflow boundary condition for finite difference lattice Boltzmann method. Transactions of the Japan Society for Computational Methods in Engineering, 6(1), pp. 7-12, 2006.

[23] Fujii, K., Numerical Method for Computational Fluid Dynamics, University of Tokyo Press: Tokyo, pp. 174-180, 1994. (In Japanese.) 